\title{
Optimization of Age Replacement Policy for Aging Multi-state System with Imperfect Maintenance
}

\author{
Yen-Luan Chen \\ Department of Marketing Management, \\ Takming University of Science and Technology, \\ Taipei, Taiwan \\ clchen@takming.edu.tw
}

\begin{abstract}
This article proposes the modified age replacement policy for a multi-state system composed of multi-state elements. The degradation of the multi-state element is assumed to follow the non-homogeneous continuous time Markov process which is a continuous time and discrete state process. A recursive approach is presented to efficiently compute the time-dependent state probability distribution of the multi-state element. The state and performance distribution of the entire multi-state system are evaluated via the combination of the stochastic process and the Lz-transform method. The concept of user-centered reliability measure is developed based on the system performance and the user demand. We develop the age replacement policy for an aging multi-state system subject to imperfect maintenance in a failure state. The optimum replacement schedule which minimizes the mean cost rate is derived analytically and discussed numerically.
\end{abstract}

Keywords-multi-state system; non-homogeneous continuous time Markov process; maintenance; optimization; reliability

\section{INTRODUCTION}

To maintain a system in a working state, avoidance of system failure during operation is important for reliability engineers. Various kinds of systems suffer deterioration due to age and use, it is reasonable to conduct maintenance activities at periodic times or after a certain age of the system. Age replacement policy (ARP) is a well-known preventive replacement policy: an operating system is replaced at age $T$ or at failure, whichever occurs first [1]. However, when a system experiences failures, it is either scrapped or minimally repaired in many practical situations. Brown and Proschan [2] considered a model in which, upon failure, the system is replaced with probability $p$ and is minimally repaired with probability $q(=1-p)$, and this activity can be called as imperfect maintenance with $(p, q)$ rule [3]. A large amount of literature models and extends the imperfect maintenance activity described above for traditional binary state systems, and some recent applications can be found in such as [4] - [5]. In this paper, we will develop the modified age replacement policy for a multi-state system (MSS) consisting of multi-state elements and incorporating imperfect maintenance quality.

In multi-state reliability modeling, the system may have more than two states of working efficiency, and the MSS should be considered thus. The MSS concerned in the present paper is defined as a system that has a finite number of discrete performance rates, from perfectly functioning to complete failure, resulting from the degradation or/and failure of some elements in the system [6]. Such a MSS is usually viewed as in a failure state once its performance rate falls below the user demand (required performance level). Therefore, the MSS reliability can be measured as the probability that the system has not reached any of the states with the performance rate less than the specified demand [7] - [8].

Many real-world systems are composed of multi-state elements (MSEs), which have different performance levels and several failure modes with various effects on the system's entire performance [9]. To describe the degradation process of individual elements, Lisnianski and Levitin [6] employ the homogeneous continuous time Markov model which assumes that the element's transition intensity to the next state only depends on the current state. However, it is more realistic to consider the case that an element's deterioration process is not only related to the current state, but also to the age effect of the element [10]. Taking this concept into account, the nonhomogeneous continuous time Markov process (NHCTMP) is utilized in this paper to derive the stochastic process of individual elements by means of considering the timedependent increasing state transition intensity.

In this paper, we treat the modified age replacement policy for an aging MSS composed of MSEs. The degradation of multi-state element (MSE) is assumed to follow the NHCTMP, and a recursive approach is presented to efficiently compute the time-dependent state probability distribution of MSE. The Lztransform method proposed in [11] and the concept of user demand are employed to facilitate the reliability assessment of series-parallel MSS consisting of MSEs. We concern with modeling the MSS and undergo imperfect maintenance with $(p, q)$ rule when the performance of MSS degrades to a failure state. The optimum replacement time $T^{*}$ for the maintenance policy can be determined by minimizing the expected cost per unit time.

\section{MSS DESCRIPTION AND RELIABILITY MEASURES}

\section{A. System Description}

Suppose that a multi-state system (MSS) consists of $n$ independent multi-state elements (MSEs). Element $k$ in MSS 
can have $m_{k}+1$ different states corresponding to the performance rates, represented by the set $\left\{G_{k, 0}, G_{k, 1}, \cdots, G_{k, m_{k}}\right\}$, where $G_{k, j}$ is the performance rate in the state $j$, $j \in\left\{0,1, \cdots, m_{k}\right\}$. Let $\Phi_{k}(t)$ and $G_{k}(t)$ be the state and performance rate of element $k$ at time $t$, respectively, where $\Phi_{k}(t) \in\left\{0,1, \cdots, m_{k}\right\}$ and $G_{k}(t) \in\left\{G_{k, 0}, G_{k, 1}, \cdots, G_{k, m_{k}}\right\}$. The degradation of element $k$ from the best state (perfect functioning) $\Phi_{k}(0)=m_{k}$ to lower states $\Phi_{k}(t) \in\left\{m_{k}-1\right.$, $\left.m_{k}-2, \cdots, 0\right\}$ is modeled using a general stochastic process known as a non-homogeneous continuous time Markov process (NHCTMP), where state 0 is the worst state (complete failure). We also assume that the process of element $k$ can be degraded directly to any lower state from state $i$ to state $j$ for all $j<i$ and $i, j \in\left\{m_{k}, m_{k}-1, \cdots, 0\right\}$.

For element $k$, the instantaneous transition rate of from state $i$ to state $j$ at time $t$ can be written as

$$
\lambda_{i, j}^{k}(t) \equiv \lim _{h \rightarrow 0} \frac{\operatorname{Pr}\left\{\Phi_{k}(t+h)=j \mid \Phi_{k}(t)=i\right\}}{h}
$$

A typical state-space diagram of a MSE $k$ is illustrated in Figure1.

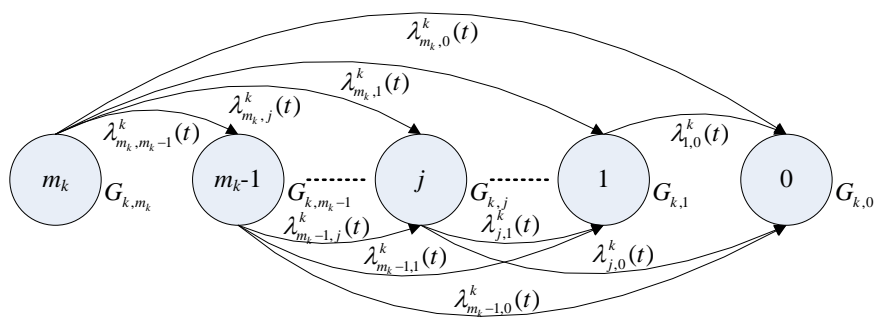

Figure 1. MSS structure and state-space diagrams for the system's elements.

Let $P_{k, j}(t) \equiv \operatorname{Pr}\left\{\Phi_{k}(t)=j\right\} \quad$ be the element state probability in state $j$ at time $t$, and $P_{k, j}^{\prime}(t) \equiv \mathrm{d} P_{k, j}(t) / \mathrm{d} t$, where $j \in\left\{m_{k}, m_{k}-1, \cdots, 0\right\}$. The state probabilities satisfy the condition $\sum_{j=0}^{m_{k}} P_{k, j}(t)=1$, because at any instant $t$, element $k$ can always be in one and only one of $m_{k}+1$ states.

The Chapman-Kolmogorov (C-K) forward equations for element $k$ in MSS following a NHCTMP can be written in the matrix form

$$
\begin{aligned}
& {\left[P_{k, m_{k}}^{\prime}(t) P_{k, m_{k}-1}^{\prime}(t) P_{k, m_{k}-2}^{\prime}(t) \cdots P_{k, 1}^{\prime}(t) P_{k, 0}^{\prime}(t)\right]} \\
& =\left[\begin{array}{cccccc}
\lambda_{m_{k}, m_{k}}^{k}(t) & \lambda_{m_{k}, m_{k}-1}^{k}(t) & \lambda_{m_{k}, m_{k}-2}^{k}(t) & \cdots & \lambda_{m_{k}, 1}^{k}(t) & \lambda_{m_{k}, 0}^{k}(t) \\
0 & \lambda_{m_{k}-1, m_{k}-1}^{k}(t) & \lambda_{m_{k}-1, m_{k}-2}^{k}(t) & \cdots & \lambda_{m_{k}-1,1}^{k}(t) & \lambda_{m_{k}-1,0}^{k}(t) \\
0 & 0 & \lambda_{m_{k}-2, m_{k}-2}^{k}(t) & \cdots & \lambda_{m_{k}-2,1}^{k}(t) & \lambda_{m_{k}-2,0}^{k}(t) \\
\vdots & \vdots & \vdots & \ddots & \vdots & \vdots \\
0 & 0 & 0 & \cdots & \lambda_{1,1}^{k}(t) & \lambda_{1,0}^{k}(t) \\
0 & 0 & 0 & \cdots & 0 & 0
\end{array}\right] \\
& {\left[P_{k, m_{k}}(t) P_{k, m_{k}-1}(t) P_{k, m_{k}-2}(t) \cdots P_{k, 1}(t) P_{k, 0}(t)\right],}
\end{aligned}
$$

and $\lambda_{i, i}^{k}(t)=-\sum_{j=0}^{i-1} \lambda_{i, j}^{k}(t)$. To determine the state probability $P_{k, j}(t)$, the C-K equations (2) can be recursively solved to obtain

$$
P_{k, m_{k}}(t)=\exp \left[\int_{0}^{t} \lambda_{m_{k}, m_{k}}^{k}(s) \mathrm{d} s\right]
$$

$$
\begin{array}{r}
P_{k, j}(t)=\sum_{i=j+1}^{m_{k}} \int_{0}^{t} P_{k, i}\left(\tau_{m_{k}+1-i}\right) \lambda_{i, j}^{k}\left(\tau_{m_{k}+1-i}\right) \exp \left[\int_{\tau_{m_{k}+1-i}}^{t} \lambda_{j, j}^{k}(s) \mathrm{d} s\right] \mathrm{d} \tau_{m_{k}+1-i}, \\
j=m_{k}-1, m_{k}-2, \cdots, 0,
\end{array}
$$

under the initial conditions $P_{k, m_{k}}(0)=1$ and $P_{k, j}(0)=0$ for $j=m_{k}-1, m_{k}-2, \cdots, 0$.

\section{B. MSS Reliability Measures}

The universal generating function (UGF) is a z-transformbased approach first proposed by Ushakov [12] and has been widely applied to MSS reliability analysis. A new Lz-transform method presented by Lisnianski [11] can be applied to aging MSS. Lz-transform is similar to UGF and is proven that there are existence and uniqueness for such a case where transition intensities are continuous functions of time.

By means of $L z$-transform, the entire MSS may have $M+1$ different states corresponding to the cumulative performance rates, which is unambiguously determined by the system configuration and performance rates of elements. Possible performance rates are represented by the set $\left\{G_{0}, G_{1}, \cdots, G_{M}\right\}$, where $G_{j}$ is the performance rate in the state $j \in\{0,1, \cdots, M\}$. Let $\Phi(t)$ and $G(t)$ be the state and performance rate of the system at time $t$, respectively. The probability associated with the individual system state in state $j$ at time $t$ is denoted by $P_{j}(t)\left(\equiv \operatorname{Pr}\{\Phi(t)=j\}=\operatorname{Pr}\left\{G(t)=G_{j}\right\}\right), j \in\{0,1, \cdots, M\}$.

The traditional reliability measure for a binary state system only focuses on the time to failure. When applied to the MSS, reliability can be considered to be a measure of the ability of a system to meet user demand [13]. The user of the system sets a minimum expected performance rate from the system, called the user demand, represented as $w$. During each operation 
cycle, as the system's output performance $G(t)$ at time $t$ drops below $w$, the system is interpreted to have failed from the user's perspective even though the system might not have physically failed in reality. Therefore, the general definition of MSS reliability can be given by $R(t, w) \equiv \operatorname{Pr}\{G(t) \geq w\}$. Furthermore, we define $S_{w} \equiv \inf \left\{j: G_{j} \geq w\right\}$, then the entire set of possible states can be divided into two disjoint subsets corresponding to acceptable states $\left\{S_{w}, S_{w}+1, \cdots, M\right\}$ and unacceptable (or failure) states $\left\{0,1, \cdots, S_{w}-1\right\}$. The reliability function can be rewritten as

$$
R(t, w) \equiv R\left(t, S_{w}\right) \equiv \operatorname{Pr}\left\{\Phi(t) \geq S_{w}\right\}=\sum_{j=S_{w}}^{M} P_{j}(t)
$$

Based on the same manner as in the traditional binary state system, let $X$ be the life time of a MSS, then the distribution function $F\left(t, S_{w}\right)$, the probability density function $f\left(t, S_{w}\right)$, and the failure rate $r\left(t, S_{w}\right)$ of $X$ can be also obtained according to the reliability function.

\section{AGE REPLACEMENT MODEL}

A modified age replacement policy for a MSS in which maintenances take place according to the following scheme.

A new MSS with survival, failure time distribution, and failure rate under the user demand $w$ described in Section II is installed at time 0 . A planned replacement is carried out whenever the system reaches age $T$. It is assumed that the MSS failures at age $t$ can experience two types: a type-I failure (minor) occurs with probability $q$ and is corrected by minimal repair, whereas a type-II failure (catastrophic) occurs with probability $p(=1-q)$ and requires an unplanned replacement. In summary, the MSS is replaced at age $T$ or at any type-II failure, whichever occurs first. A renewal cycle is defined as the time interval between two consecutive replacements.

The mean cost rate of a replacement cycle can be derived as

$$
J\left(T, S_{w}\right) \equiv \frac{C_{T} \bar{F}_{p}\left(T, S_{w}\right)+C_{Y} F_{p}\left(T, S_{w}\right)+C_{M} \int_{0}^{T} \bar{F}_{p}\left(t, S_{w}\right) \operatorname{qr}\left(t, S_{w}\right) \mathrm{d} t}{\int_{0}^{T} \bar{F}_{p}\left(t, S_{w}\right) \mathrm{d} t},
$$

where $\bar{F}_{p}\left(t, S_{w}\right)$ is the survival function of time to first type-II failure, $C_{Y}$ and $C_{T}$ are the unplanned and planned replacement costs. To determine the optimal $T^{*}$ that minimizes $J\left(T, S_{w}\right)$, we see that $\mathrm{d} J\left(T, S_{w}\right) / \mathrm{d} T=0$ if and only if

$$
\varphi\left(T, S_{w}\right) \int_{0}^{T} \bar{F}_{p}\left(t, S_{w}\right) \mathrm{d} t-\left[\left(C_{Y}-C_{T}\right) F_{p}\left(T, S_{w}\right)+C_{M} \int_{0}^{T} \bar{F}_{p}\left(t, S_{w}\right) \operatorname{qr}\left(t, S_{w}\right) \mathrm{d} t\right]=C_{T},
$$

where $\varphi\left(T, S_{w}\right) \equiv\left(C_{Y}-C_{T}\right) \operatorname{pr}\left(T, S_{w}\right)+C_{M} q r\left(T, S_{w}\right)$. If $\varphi\left(T, S_{w}\right)$ is increasing in $T$ and $\lim _{T \rightarrow \infty} \varphi\left(T, S_{w}\right)=\infty$, then there exists a finite and unique $T^{*}$ which satisfies (6) and the optimal mean cost rate is $J\left(T^{*}, S_{w}\right)=\varphi\left(T^{*}, S_{w}\right)$.

\section{NUMERICAL EXAMPLE}

Consider a flow transmission MSS that is shown in Figure 1 consisting of three aging MSE [14]. The water or oil flow is transmitted from left to right.

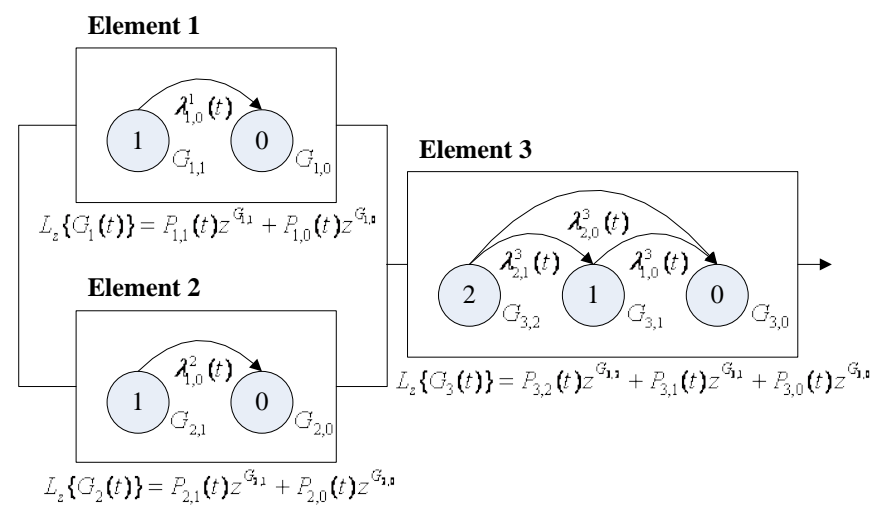

Figure 2. MSS structure and state-space diagrams for the system's elements.

Suppose that the degradation of element $k(k=1,2,3)$ follows NHCTMP with instantaneous transition rates of a Weibull process as $\lambda_{i, j}^{k}(t)=2 t^{3} /(i-j)^{4}$ for $i>j$, and the age-related transition intensities are given in Table I.

TABLE I. PARAMETERS FOR EACH ELEMENT

\begin{tabular}{c|c|c|c|c}
\hline $\begin{array}{c}\text { Element } \\
(\#)\end{array}$ & State & $\begin{array}{c}\text { Performance rates } \\
\text { (tons/min) }\end{array}$ & $\begin{array}{c}\text { Transition intensities } \\
\left(\text { year }^{-1}\right)\end{array}$ & $\begin{array}{c}\text { State probabilities and } \\
\text { initial condition }\end{array}$ \\
\hline 1 & 1 & $G_{1,1}=1.5$ & $\lambda_{1,0}^{1}(t)=2 t^{3}$ & $P_{1,1}(t), P_{1,1}(0)=1$ \\
& 0 & $G_{1,0}=0.0$ & & $P_{1,0}(t), P_{1,0}(t)=0$ \\
\hline 2 & 1 & $G_{2,1}=2.0$ & $\lambda_{1,0}^{2}(t)=2 t^{3}$ & $P_{2,1}(t), P_{2,1}(0)=1$ \\
& 0 & $G_{2,0}=0.0$ & & $P_{2,0}(t), P_{2,0}(t)=0$ \\
\hline 3 & 2 & $G_{3,2}=4.0$ & $\lambda_{2,1}^{3}(t)=2 t^{3}$ & $P_{3,2}(t), P_{3,2}(0)=1$ \\
& 1 & $G_{3,1}=1.8$ & $\lambda_{2,0}^{3}(t)=0.125 t^{3}$ & $P_{3,1}(t), P_{3,1}(0)=0$ \\
& 0 & $G_{3,0}=0.0$ & $\lambda_{1,0}^{3}(t)=2 t^{3}$ & $P_{3,0}(t), P_{3,0}(0)=0$ \\
\hline
\end{tabular}

Further, the state probability of each element can be recursively solved corresponding C-K equation (2) and directly determined by using equations (3) and (4) as below.

- $\quad$ For element $1, P_{1,1}(t)=e^{-0.5 t^{4}}$ and $P_{1,0}(t)=1-e^{-0.5 t^{4}}$.

- For element 2, $P_{2,1}(t)=e^{-0.5 t^{4}}$ and $P_{2,0}(t)=1-e^{-0.5 t^{4}}$.

- For element 3,

$$
\begin{aligned}
& P_{3,2}(t)=e^{-0.7391 t^{4}}, \quad P_{3,1}(t)=16 e^{-0.5 t^{4}}-16 e^{-0.7391 t^{4}}, \quad \text { and } \\
& P_{3,0}(t)=1-16 e^{-0.5 t^{4}}+15 e^{-0.7391 t^{4}} .
\end{aligned}
$$

The system output performance rate is defined as the maximum flow that can be transmitted. According to the 
system structure $\phi$ and performance rate combination property, the output performance rate of the entire MSS is defined as $G(t)=\phi\left(G_{1}(t), G_{2}(t), G_{3}(t)\right)=\min \left\{G_{1}(t)+G_{2}(t), G_{3}(t)\right\}$. Then the Lz-transform of the entire MSS is

$L_{z}\{G(t)\}=\sum_{j_{1}=0}^{1} \sum_{j_{2}=0}^{1} \sum_{j_{3}=0}^{2}\left(P_{1, j_{1}}(t) P_{2, j_{2}}(t) P_{3, j_{3}}(t) z^{\min \left(G_{1, j_{1}}+G_{2, j_{2}}, G_{3, j_{3}}\right)}\right)=\sum_{j=0}^{4} P_{j}(t) z^{G_{j}}$,

where $G_{j}$ and $P_{j}(t)$ as shown in Table II

TABLE II. PERFORMANCE RATES AND STATE PROBABILITIES OF MSS

\begin{tabular}{c|c|l}
\hline State & $\begin{array}{c}\text { Performance rates } \\
\text { (tons/min) }\end{array}$ & \multicolumn{1}{|c}{ State probabilities } \\
\hline 4 & $G_{4}=3.5$ & $P_{4}(t)=P_{1,1}(t) P_{2,1}(t) P_{3,2}(t)$ \\
3 & $G_{3}=2.0$ & $P_{3}(t)=P_{1,0}(t) P_{2,1}(t) P_{3,2}(t)$ \\
2 & $G_{2}=1.8$ & $P_{2}(t)=P_{2,1}(t) P_{3,1}(t)$ \\
1 & $G_{1}=1.5$ & $P_{1}(t)=P_{1,1}(t) P_{2,0}(t)\left[P_{3,1}(t)+P_{3,2}(t)\right]$ \\
0 & $G_{0}=0.0$ & $P_{0}(t)=P_{1,0}(t) P_{2,0}(t)+P_{1,1}(t) P_{3,0}(t)+P_{1,0}(t) P_{2,1}(t) P_{3,0}(t)$ \\
\hline
\end{tabular}

Assuming the user demand $w=1.8$ (tons/min), then $S_{w}=S_{1.8}=2$, the acceptable states are $\{2,3,4\}$, and failure states are $\{0,1\}$. Therefore, the reliability function $R\left(t, S_{w}\right)$ under the user demand $w=1.8$ is equal to $P_{2}(t)+P_{3}(t)+P_{4}(t)$.

We consider a repair limit replacement policy where, in a failure state, one replaces the system or repairs it depending on the random cost $C$ with density function $l(u)$. A system undergoes a corrective replacement if $C>\delta c_{\infty}$ and is minimally repaired if $C \leq \delta c_{\infty}$, where $c_{\infty}$ is the constant cost of replacement at failure and $\delta(0 \leq \delta \leq 1)$ can be interpreted as a fraction of the constant cost $c_{\infty}$. Hence, $\delta$ satisfies $q=\int_{0}^{\delta c_{\infty}} l(u) \mathrm{d} u$, and the expected minimal repair cost $c_{m}$ can be given by $c_{m}=\int_{0}^{\delta c_{\infty}} u l(u) \mathrm{d} u / q$. The parameter $q$ is varied to determine its influence on the optimal policy and its mean cost. The optimal age replacement times and related minimum mean cost rates for the MSS with imperfect maintenance are reported in Tables III.

TABLE III. OPTIMAL POLICY AND MEAN COST RATE FOR A MSS $C_{T}=1000, C_{Y}=1500, C_{\infty}=1000, C \sim N\left(300,75^{2}\right), w=1.5,1.8$

\begin{tabular}{cccccc}
\hline & \multicolumn{2}{c}{ Case 1. $w=1.5$} & & \multicolumn{2}{c}{ Case 2. $w=1.8$} \\
\cline { 2 - 3 } \cline { 5 - 6 } & $T^{*}$ & $J\left(T^{*}, S_{1.5}\right)$ & & $T^{*}$ & $J\left(T^{*}, S_{1.8}\right)$ \\
\hline 1.0 & 1.1320 & 1052.3598 & & 1.0998 & 1168.3735 \\
0.9 & 1.1283 & 1059.6843 & & 1.0966 & 1182.4403 \\
0.8 & 1.1213 & 1069.2934 & & 1.0895 & 1200.5788 \\
0.7 & 1.1131 & 1080.3719 & & 1.0806 & 1219.7508 \\
0.6 & 1.1040 & 1091.3448 & & 1.0706 & 1239.5490 \\
0.5 & 1.0995 & 1127.8942 & & 1.0599 & 1259.9358 \\
0.4 & 1.0846 & 1114.5013 & & 1.0487 & 1280.7967 \\
0.3 & 1.0744 & 1125.8154 & & 1.0370 & 1301.6417 \\
0.2 & 1.0641 & 1137.9952 & & 1.0248 & 1322.4599 \\
0.1 & 1.0533 & 1149.6630 & & 1.0121 & 1344.1460 \\
0.0 & 1.0415 & 1163.0153 & & 0.9978 & 1367.3369 \\
\hline
\end{tabular}

From the numerical results, we have the following observations:

- When the probability of minimal repair increases, we should extend the replacement schedule in order to reduce the minimum mean cost rates.

- By comparing Case 1 and Case 2, if the user demand decreases, it is observable that the replacement schedule should be extended via the improved operating cost rate.

- We see that some improvement can be made in the minimum mean cost rate if one allows for minimal repair at minor failure.

\section{ACKNOWLEDGMENT}

This research was supported by the National Science Council of Taiwan, ROC, under Grant No. NSC102-2410-H147-008.

\section{REFERENCES}

[1] R.E. Barlow and L.C. Hunter, "Optimum preventive maintenance policies,” Operations Research, vol. 8, pp. 90-100, 1960.

[2] M. Brown and F. Proschan, "Imperfect repair," Journal of Applied Probability, vol. 20, pp. 851-859, 1983.

[3] H. Pham and H. Wang, "Imperfect maintenance,” European Journal of Operational Research, vol. 94, pp. 425-438, 1996.

[4] C.C. Chang, S.H. Sheu, and Y.L. Chen, "Optimal number of minimal repairs before replacement based on a cumulative repair-cost limit policy,” Computers \& Industrial Engineering, vol. 59, pp. 603-610, 2010.

[5] Y.L. Chen, “A bivariate optimal imperfect preventive maintenance policy for a used system with two-type shocks," Computers \& Industrial Engineering, vol. 63, pp. 1227-1234, 2012.

[6] A. Lisnianski and G. Levitin, Multi-State System Reliability, Assessment, Optimization and Application, World Scientific: Singapore, 2003.

[7] J. Xue and K. Yang, "Dynamic reliability analysis of coherent multistate systems,” IEEE Transactions on Reliability, vol. 44(4), pp. 683-688, 1995.

[8] Y.W. Liu and K.C. Kapur, "Reliability measures for dynamic MultiState systems and their applications for system design and evaluation,” IIE Transactions, vol. 38(6), pp. 511-520, 2006.

[9] A. Lisnianski, "Extended block diagram method for a multi-state system reliability assessment,” Reliability Engineering and System Safety, vol. 92(12), pp. 1601-1607, 2007.

[10] Y.W. Liu and K.C. Kapur, “Customer's cumulative experience measures for reliability of non-repairable aging multi-state systems,” Quality Technology \& Quantitative Management, vol. 4(2), pp. 225-234, 2007.

[11] A. Lisnianski, Lz-transform for a Discrete-state Continuous-time Markov Process and its Applications to Multi-state System Reliability, In: Recent Advance in System Reliability. Signatures, Multi-state Systems and Statistical Inference, Springer: London, 2012.

[12] I. Ushakov, "Universal generating function," Soviet Journal of Computing System Science, vol. 24(5), pp. 118-129, 1986.

[13] G. Levitin and A. Lisnianski, "Optimization of imperfect preventive maintenance for multi-state systems,” Reliability Engineering and System Safety, vol. 67, pp. 193-203, 2000.

[14] C.M. Tan and N. Raghavan, "A framework to practical predictive maintenance modeling for multi-state systems,” Reliability Engineering \& System Safety, vol. 93(8), pp. 1138-1150, 2008. 